\title{
Low Dose High-Resolution Electron Microscopy (HREM) of Poly(3,4-Ethylene Dioxythiophene) (PEDOT) Films
}

\author{
Jinghang $\mathrm{Wu}^{*}$ and David C. Martin ** \\ * Macromolecular Science and Engineering, University of Michigan, Ann Arbor, MI 48109 \\ ** Materials Science and Engineering, University of Delaware, Newark, DE 19716; Materials \\ Science and Engineering, Macromolecular Science and Engineering and Biomedical Engineering \\ University of Michigan, Ann Arbor, MI 48109 (milty@udel.edu)
}

Poly(3,4-ethylene dioxythiophene) (PEDOT or PEDT) is a widely used conjugated polymer of interest for a variety of applications. The rigid backbone conformation of PEDOT facilitates charge transport and favors crystallization that reduces its solubility and processability. PEDOT has an orthorhombic crystal structure with typical lattice parameters a, b and c of $1.4 \mathrm{~nm}, 0.68$ $\mathrm{nm}$ and $0.78 \mathrm{~nm}$ respectively. The solid-state structure is relatively limited; PEDOT films have a relatively low degree of crystallinity. Also the d100 spacings for a given PEDOT sample range from 1.15 to $1.52 \mathrm{~nm}$ depending on the counterions used during deposition [1]. Studies of the local packing and organization of polymers and organic molecules into ordered assemblies using low dose HREM is an ongoing topic of interest in our research group [2].

A commercially available PEDOT associated with poly(styrene sulfonate) counterion (PSS) $\left(\right.$ Baytron $^{\circledR} \mathrm{P}$ ) was studied before and after annealing at $150 \mathrm{C}$. HREM images were obtained using a $300 \mathrm{kV}$ JEOL 3000EX. The critical dose of PEDOT has been estimated to be $0.1 \mathrm{C} / \mathrm{cm} 2$. The (100) lattice spacing for PEDOT-PSS has been estimated to be $1.4 \mathrm{~nm}$ from HREM images (FIG 1). Under certain conditions we have also been able to obtain low dose HREM images of the $(020) \pi-\pi$ stacking distance in PEDOT before and after annealing. These $(020)$ fringes show an increase from $0.34 \mathrm{~nm}$ (as cast) (FIG 2) to $0.39 \mathrm{~nm}$ after annealing at $150{ }^{\circ} \mathrm{C}$ (FIG 3). These observations are generally consistent with previous studies showing an expansion in the lateral packing of PEDOT on heating. PEDOT chains stack along $b$-axis forming face to face layers with a typical $\pi-\pi$ stacking distance of $b / 2$. 


\section{References}

[1] D. C. Martin et al., Polymer Reviews, 50, (2010), 340.

[2] D.C. Martin et al., Journal of Polymer Science: Physics, 43, (2005). 1749.

[3] J. Lee et al., Nano Lett., 8, (2008), 2315.

[4] This research was supported by the National Science Foundation (NSF).

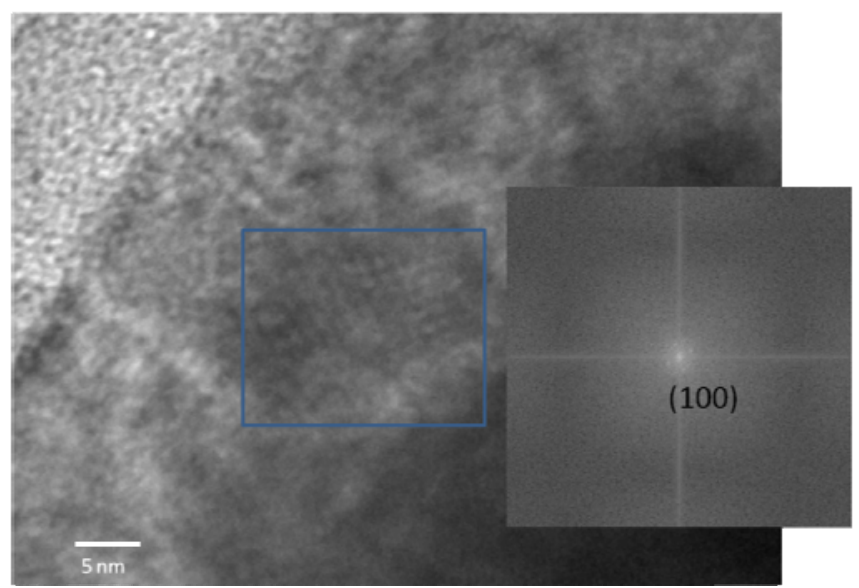

FIG. 1. Low dose HREM images of PEDOT PSS in Baytron ${ }^{\circledR} \mathrm{P}$ with an embedded digital FFT showing $1.4 \mathrm{~nm}$ (100) fringes.

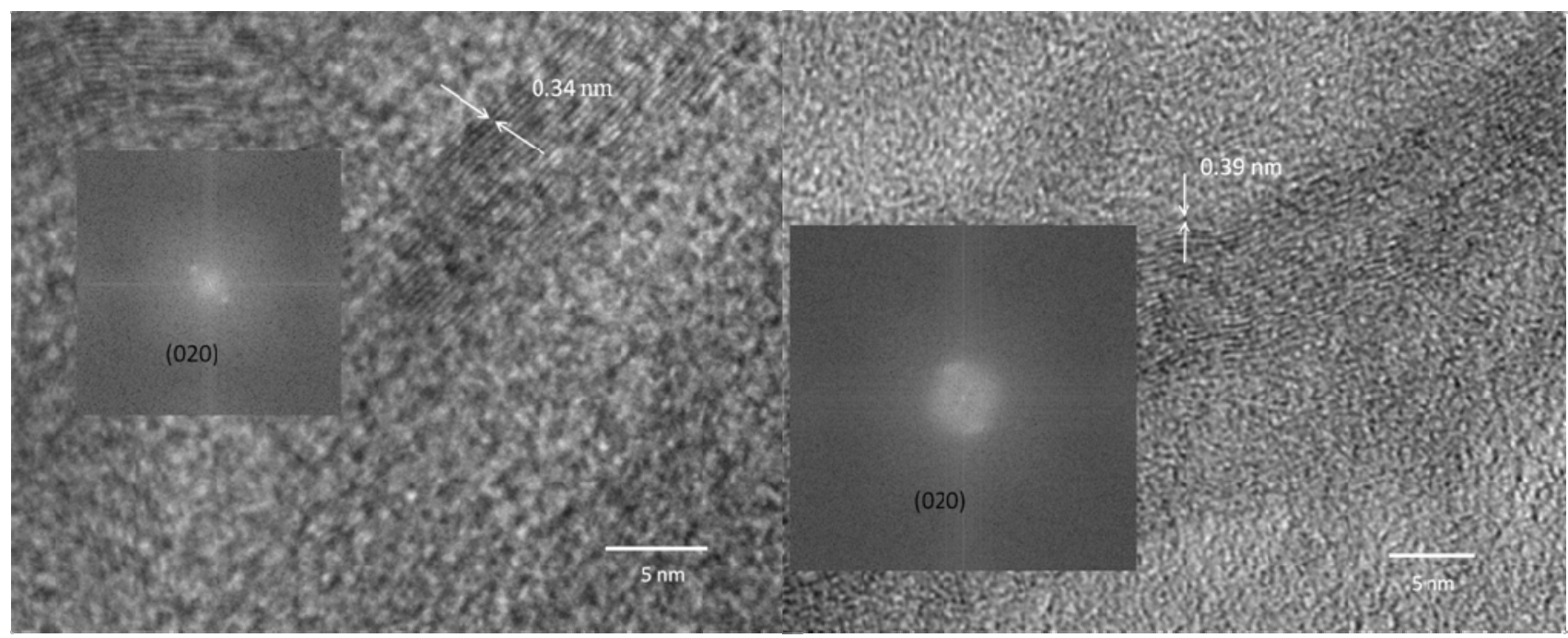

FIG. 2. Low dose High resolution TEM image of Baytron $® P$ showing (020) fringes corresponding to $\pi-\pi$ stacking before (left) and after (right) annealing at $150 \mathrm{C}$. FFT analysis indicates a relatively small amount of local misorientation. 